\title{
Anhedonia is associated with poor health status and more somatic and cognitive symptoms in patients with coronary artery disease
}

\author{
Aline J. Pelle • Susanne S. Pedersen - Ruud A. M. Erdman • \\ Marten Kazemier • Marquita Spiering • \\ Ron T. van Domburg • Johan Denollet
}

Accepted: 1 November 2010/Published online: 17 November 2010

(C) The Author(s) 2010. This article is published with open access at Springerlink.com

\begin{abstract}
Purpose The effectiveness of cardiac rehabilitation (CR) in patients with coronary artery disease (CAD) is moderated by negative emotions and clinical factors, but no studies evaluated the role of positive emotions. This study examined whether anhedonia (i.e. the lack of positive affect) moderated the effectiveness of CR on health status and somatic and cognitive symptoms.

Methods CAD patients $(n=368)$ filled out the Hospital Anxiety and Depression Scale (HADS) to assess anhedonia at the start of CR, and the Short-Form Health Survey (SF-36) and the Health Complaints Scale (HCS) at the start of $\mathrm{CR}$ and at 3 months to assess health status and somatic and cognitive symptoms, respectively.

Results Adjusting for clinical and demographic factors, health status improved significantly during the follow-up $(F(1,357)=10.84, P=.001)$. Anhedonic patients reported poorer health status compared with non-anhedonic patients, with anhedonia exerting a stable effect over time
\end{abstract}

A. J. Pelle · S. S. Pedersen $(\bowtie) \cdot$ J. Denollet

Center of Research on Psychology in Somatic Diseases

(CoRPS), Department of Medical Psychology, Tilburg

University, PO Box 90153, 5000, LE, Tilbur, The Netherlands

e-mail: s.s.pedersen@uvt.nl

S. S. Pedersen · R. A. M. Erdman · R. T. van Domburg Department of Cardiology, Thoraxcenter, Erasmus Medical Center, Rotterdam, The Netherlands

M. Kazemier · M. Spiering

Rotterdam Organization for Cardiac Rehabilitation,

Rotterdam, The Netherlands

R. A. M. Erdman

Department of Medical Psychology and Psychotherapy,

Erasmus Medical Center, Rotterdam, The Netherlands
$(F(1,358)=34.80, \quad P<.001)$. Somatic and cognitive symptoms decreased over time $(F(1,358)=3.85, P=.05)$. Anhedonics experienced more benefits in terms of somatic and cognitive symptoms over time $(F(1,358)=13.00$, $P<.001)$.

Conclusion Anhedonic patients reported poorer health status and higher levels of somatic and cognitive symptoms prior to and after CR. Somatic and cognitive symptoms differed as a function of anhedonia over time, but health status did not. Anhedonia might provide a new avenue for secondary prevention in CAD.

Keywords Anhedonia - Cardiac rehabilitation . Health complaints - Health status - Positive affect

\section{Introduction}

Cardiac rehabilitation (CR) has shown to be effective in improving clinical outcome [1] and health status [2], and in reducing mortality [3] in patients with established coronary artery disease (CAD). However, the effectiveness of $\mathrm{CR}$ seems to be moderated by both clinical and psychological factors [4, 5]. Regarding psychological factors, most attention has been paid to the detrimental effects of negative affect, such as depression [6], anxiety [7], and general distress [8], thereby neglecting the role of positive affect. Positive affect can be described in terms of joy, cheerfulness, and happiness [9]. Importantly, positive and negative affect are not merely opposite ends of a continuum [10], with the possibility that both types of affect can be present simultaneously.

In the general cardiovascular literature, there is an increased interest in the role of positive affect on clinical outcomes. In a recent study, emotional vitality, a concept 
that is closely related to positive affect, was protective against incident CAD [11]. In patients with established $\mathrm{CAD}$, a positive relation was found between happiness and a decrease in the number of hospital readmissions after 90 days [12]. To date, studies on the relationship between positive affect-related constructs and survival have shown inconsistent results [13-17]. Anhedonia (i.e. the lack of positive affect) has been shown to independently predict the combined endpoint of adverse clinical events and mortality 1 year after an acute coronary syndrome [17] and in patients following implantation of coronary-artery stents [18], even after controlling for clinical depression and severity of depressive symptoms [17].

Apart from clinical outcomes, studies on patient-centered outcomes have been advocated, as these may help bridge the gap between research and clinical outcome [19]. To date, no studies have focused on anhedonia and patientcentered outcomes in cardiac patients. Hence, the objective of the current study was to prospectively examine the associations between anhedonia and patient-centered outcomes, defined as health status and somatic and cognitive symptoms, in CAD patients attending $\mathrm{CR}$.

\section{Method}

Study design and participants

Consecutive patients with CAD $(n=368$; response rate $=71.2 \%$ ) referred to CAPRI (cardiac rehabilitation program at the Rotterdam Organization for Cardiac Rehabilitation, Rotterdam, The Netherlands) between March 2004 and October 2005, participating in the identification of subgroups of HEART patients that may not benefit optimally from CArdiac Rehabilitation (HEARTCARE) study, comprised the sample for the current study. Patients diagnosed with chronic heart failure by their treating cardiologist (due to participation in another study within the institution) and those with insufficient knowledge of the Dutch language to be able to complete questionnaires were excluded from participation. A flow-chart of the patient selection is provided in Fig. 1. Patients were asked to complete a set of psychological questionnaires at baseline and at 3-month follow-up (i.e. prior to and after completion of the rehabilitation program). The present study was set up as a between-subjects design, and not as a randomized controlled trial, given that $\mathrm{CR}$ has been shown to decrease mortality. Hence, it would be unethical to withhold CR from patients [6].

The study protocol was approved by the medical ethics committee of the Erasmus Medical Center, Rotterdam. The study was conducted according to the Helsinki Declaration and every patient provided written informed consent.

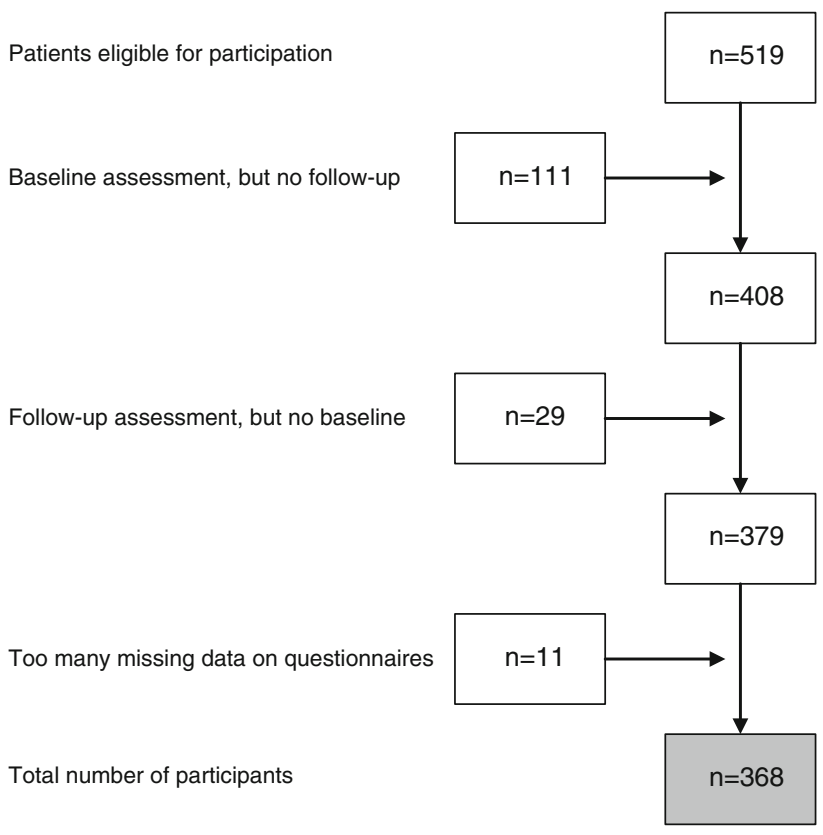

Fig. 1 Flow chart of patient selection

\section{Contents of the rehabilitation program}

The program was multi-factorial comprising an introduction module, a physical exercise component, modules on risk factors, stress management, diet, medication, weight loss, and smoking cessation, with the possibility of having individual counseling by a psychiatrist, psychologist, or social worker if necessary.

\section{Materials}

\section{Demographic and clinical variables}

Demographic variables included gender, age, educational level, marital status, and employment status. Clinical variables, obtained from the patients' medical records, included initial diagnosis, cardiac history prior to the referral event (i.e. previous myocardial infarction (MI), coronary artery bypass graft surgery (CABG), or percutaneous coronary intervention (PCI)), prescribed medications, smoking, and risk factors (i.e. hypercholesterolemia, hypertension, diabetes, family history, renal insufficiency, chronic obstructive pulmonary disease (COPD), and liver insufficiency).

\section{Anhedonia}

Anhedonia (i.e. the lack of positive affect) was assessed with the Hospital Anxiety and Depression Scale (HADS) at baseline [20, 21]. Items are scored on a 4-point Likert scale ranging from 0 to 3 . Previous research using factor analysis 
of the HADS has identified a psychometrically sound positive subscale in patients with CAD [18]. In line with this study, the Positive Affect scale was computed by summing up four items (i.e. items 2, 4, 12, and 6) (range $[0-12]$, mean $=9.79 \pm 2.54$ ) [18]. Anhedonia was defined as a score $\leq 7$ on the Positive Affect scale (i.e. one SD below the mean). The mean scores on the Negative Affect (range [0-12], mean $=3.08 \pm 2.56$ ) and the Relaxed Affect scale (range [0-9], mean $=6.03 \pm 2.30$ ) were in line with this study as well [18].

In order to cross-validate the three subscales derived from the HADS, patients also filled out the Global Mood Scale (GMS) to assess Positive and Negative Affect [22-24]. The 20 items are answered on a 5-point Likert scale ranging from 0 ('not at all') to 4 ('extremely'). Both subscales have good internal consistency $(\alpha=.94$ and $\alpha=.93$, respectively) [24], and test-retest reliability over a 3 -month period for both subscales is adequate (Negative Affect $=0.66$ and Positive Affect $=0.57$ ) [22]

\section{Health status}

The Dutch version of the Short-Form Health Survey (SF-36) was administered both at baseline and at 3 months to assess generic health status [25, 26]. All 36 items are answered according to standardized response choices and contribute to eight subscales: Physical Functioning, Social Functioning, Role Limitations due to Physical Functioning, Role Limitations due to Emotional Functioning, Mental Health, Vitality, Bodily Pain, and General Health. Raw scores were transformed to summary scores according to standard scoring procedures. Subscale scores range from 0 to 100 , with higher scores indicating better levels of functioning. In addition, by using scoring algorithms, these eight scales can be summarized in two component scores: the Mental Component Score (MCS) and the Physical Component Score (PCS) [27]. The validity and reliability of the SF-36 are good [26].

\section{Somatic and cognitive symptoms}

The 24-item Health Complaints Scale (HCS), a diseasespecific measure, was administered to assess somatic and cognitive symptoms at baseline and at 3-month follow-up [28]. Both the somatic and the cognitive complaints scale consist of 12 items that are scored on a 4-point Likert scale ranging from 0 ('not at all') to 4 ('extremely'), with a score range from 0 to 48 . A high score on both subscales indicates more symptoms. Internal consistency is good $(.89<\alpha<.91)$ and test-retest reliability has proven to be adequate $(r=.69)$ in patients with established CAD [28, 29]. This instrument has been shown to be responsive to tap treatment-related changes following CR [30].
Statistical analyses

Principal Component Analysis (PCA) with varimax rotation was used to explore the factorial structure of the HADS. The scree plot was used as a criterion for the number of factors to be extracted. KMO and Bartlett's test of sphericity were used as fit-indices. KMO (.92) and Bartlett's test of sphericity $\left(\chi^{2}(91)=1966, P<.001\right)$ indicated that the data were appropriate for carrying out a PCA. The construct validity of the HADS scales (i.e. Positive Affect, Negative Affect, and Relaxed Affect) was determined by computing Pearsons' product-moment correlations between the GMS Positive and Negative Affect scales. Discrete variables were compared with the Chi-square test and continuous variables with Student's t-test for independent samples. Analyses were adjusted for multiple comparisons by means of Bonferroni corrections ( $\alpha$ /number of comparisons). To examine differences in health status between anhedonic and nonanhedonic patients prior to and after CR, we used multivariable analysis of variance (MANOVA) in order to adjust for multiple comparisons, given that the SF-36 comprises two summary scores (PCS and MCS) and the HCS comprises two subscales (somatic and cognitive symptoms). Multivariable analysis of covariance (MANCOVA) was performed to adjust for the effect of potential confounders on the relationship between anhedonia and health status and somatic and cognitive symptoms. A priori [31], we decided to include age, gender, comorbidity (COPD, diabetes, or renal insufficiency), the use of nitrates, antidepressant and anxiolytic medications, and receiving individual counseling as covariates in the analyses based on the literature [7, 32, 33]. In addition, we added the derived HADS Negative Affect scale in order to control statistically for a measure of negative affect, also to investigate whether negative and positive affect are merely the opposites of two ends of a continuum [10], or whether they can be present at the same time. This approach is in line with previous studies on the effects of anhedonia in cardiac patients $[17,18]$. Post hoc paired samples t-tests were conducted to determine differences in summary scores for the SF-36 and scale scores of the HCS for anhedonic and non-anhedonic patients separately.

A post hoc power analysis showed that with an assumed effect-size of $.20, \alpha=.05$, and a power of $95 \%$, a sample size of 327 patients was required to detect statistically significant differences in SF-36 component scores and HCS scores pre- and post-CR (repeated measures design, withinbetween interaction). This assumption was met, since analyses were performed on 368 patients. All statistical tests were two-tailed, and $P<.05$ was used to indicate statistical significance. Statistical analyses were performed using SPSS 17.0 for Windows (SPSS Inc., Chicago, Illinois, USA). 


\section{Results}

\section{Patient characteristics}

Completers ( $n=368)$ and non-completers $(n=151)$ of the CR program did not differ on baseline demographic and clinical characteristics.

The prevalence of anhedonia was $20.1 \%$ in the current sample. Patient baseline characteristics stratified by anhedonia are presented in Table 1. Differences emerged between anhedonic and non-anhedonic patients on baseline demographic and clinical characteristics, with anhedonic patients more frequently smoking $(P=.002)$ and taking more often anti-depressant and anxiolytic medications ( $P<.001$ and $P=.007$, respectively) compared with nonanhedonic patients. In addition, trends were found for nonanhedonic patients being more likely to have a partner and less likely to have individual counseling as part of CR.

\section{Characteristics of the HADS Positive Affect scale}

PCA confirmed the previously identified three-factor structure [18], comprising Positive Affect (4 items), Negative Affect (4 items), and Relaxed Affect (3 items) (Table 2). The items 8 ("I feel as if I am slowed down"), 9 ("I get a sort of frightened feeling like 'butterflies' in the stomach"), and 10 ("I have lost interest in my appearance”) were excluded from further analyses, because these items loaded diffusely on the three factors (high crossloadings), had relatively low item-correlations, and did not contribute to the internal consistency of any of the scales.

The derived HADS Positive Affect subscale showed a significant correlation with the GMS Positive Affect subscale $(r=.53, P<.001)$ and a significant negative correlation with the GMS Negative Affect subscale ( $r=$ $-.47, P<.001$ ), confirming the construct validity of this derived HADS subscale. In addition, the derived HADS Negative Affect subscale was significantly positively correlated with the GMS Negative Affect scale $(r=.50$, $P<.001)$ and negatively correlated with the GMS Positive Affect subscale $(r=-.38, P<.001)$. The HADS Relaxed Affect subscale showed significant correlations with both of the GMS Positive Affect and Negative Affect scales ( $r=.36$ and $r=-38$, both $P$ s $<.001$, respectively).

Anhedonia as a determinant of health status

MANOVA with repeated measures demonstrated a significant within-subjects effect for time $(F(1,366)=222.63$, $P<.001)$, indicating that health status improved following $\mathrm{CR}$. The time by Anhedonia interaction effect was not significant, indicating that Anhedonia had a stable effect on health status over time $(F(1,366)=1.33, P=.25)$.
However, anhedonic patients reported significantly poorer health status than non-anhedonic patients $(F(1,366)=$ 64.53, $P<.001)$. Mean scores on health status pre- and post-CR stratified by anhedonia are presented in Fig. 2.

MANCOVA showed a main effect for time $(F(1,357)$ $=10.84, P=.001)$, indicating that the overall improvement in health status after $\mathrm{CR}$ as seen in unadjusted analysis remained when correcting for potential confounders. Furthermore, a trend was found for the interaction effect for time by anxiolytic medications, denoting that patients using anxiolytic medications reported impaired health status pre- and post-CR compared with patients not using anxiolytic medications $(F(1,357)=3.45, P=.06)$. Comorbidity, age, gender, smoking, nitrate use, use of antidepressants, individual counseling, and the HADS Negative Affect scale did not interact with CR (all $P$ S $>.05)$. The time by anhedonia interaction effect was not significant, indicating that anhedonia had a stable effect on health status over time $(F(1,357)=.23, P=.63)$. The between-subjects effect for anhedonia remained significant, showing that anhedonic and non-anhedonic patients differed on self-reported health status $(F(1,357)=34.80$, $P<.001)$. The main effects for age $(F(1.357)=3.85, P=$ $.05)$, comorbidity $(F(1,357)=4.82, P=.03)$, the HADS Negative Affectivity scale $(F(1,357)=98.24, P<.001)$, and individual counseling $(F(1,357)=3.90, \quad P=.05)$ were significant.

Paired samples t-tests showed that anhedonic patients reported improvements on both the MCS and the PCS $(t(73)=-5.51$ and $t(73)=-5.21$, both $P s<.001)$. Likewise, non-anhedonic patients reported improvements on the MCS and the PCS $(t(293)=-7.52$ and $t(293)=$ -12.48 , both $P$ s $<.001)$.

Anhedonia as a determinant of somatic and cognitive symptoms

MANOVA with repeated measures yielded a significant main within-subjects effect for time, indicating a decrease in somatic and cognitive symptoms following $\mathrm{CR}$ $(F(1,366)=96.11, P<.001)$. The interaction effect for time by anhedonia was significant, showing that anhedonia did not exert a stable effect over time on somatic and cognitive symptoms $(F(1,366)=11.79, P<.001)$. Finally, the between-subjects main effect for anhedonia was significant, denoting that anhedonic and non-anhedonic patients reported different levels of somatic and cognitive symptoms $(F(1,366)=94.59, P<.001)$. Mean scores on somatic and cognitive symptoms pre- and post-CR, stratified by anhedonia, are presented in Fig. 3.

In repeated measures MANCOVA, the main effect for time was significant, indicating that after controlling for 
Table 1 Demographic and clinical data stratified by anhedonia ${ }^{a}$

\begin{tabular}{|c|c|c|c|c|}
\hline & Total sample $(n=368)$ & Anhedonia $(n=74)$ & No anhedonia $(n=294)$ & $P$ \\
\hline \multicolumn{5}{|l|}{ Socio-demographics } \\
\hline Males & $290(78.8)$ & $58(78.4)$ & $232(78.9)$ & .92 \\
\hline Age, mean (SD) & $58.0(10.2)$ & $57.7(10.1)$ & $58.1(10.3)$ & .77 \\
\hline Having a partner & $330(89.7)$ & $62(83.8)$ & $268(91.2)$ & .06 \\
\hline \multicolumn{5}{|l|}{ Clinical variables } \\
\hline Cardiac event prior to index event ${ }^{\mathrm{b}}$ & $299(81.3)$ & $60(81.1)$ & $239(81.3)$ & .97 \\
\hline Diabetes Mellitus & $54(14.7)$ & $13(17.6)$ & $41(13.9)$ & .43 \\
\hline Dyslipidemia & $185(50.3)$ & $42(56.8)$ & $143(48.6)$ & .21 \\
\hline Hypertension & $122(33.2)$ & $27(36.5)$ & $95(32.3)$ & .50 \\
\hline $\mathrm{COPD}^{\mathrm{c}}$ & $28(7.6)$ & $6(8.1)$ & $22(7.5)$ & .86 \\
\hline Currently smoking & $32(8.7)$ & $13(17.6)$ & $19(6.5)$ & $.002^{\mathrm{d}}$ \\
\hline \multicolumn{5}{|l|}{ Medication } \\
\hline ACE-inhibitors & $215(58.4)$ & $47(63.5)$ & $168(57.1)$ & .32 \\
\hline Calcium-antagonists & $42(11.4)$ & $5(6.8)$ & $37(12.6)$ & .16 \\
\hline Nitrates & $118(32.1)$ & $15(20.3)$ & $103(35.0)$ & $.02 *$ \\
\hline Statins & $298(81.0)$ & $62(83.8)$ & $236(80.3)$ & .49 \\
\hline Aspirin & $277(75.3)$ & $57(77.0)$ & $220(74.8)$ & .70 \\
\hline Diuretics & $66(17.9)$ & $10(13.5)$ & $56(19.0)$ & .27 \\
\hline Anti-depressants & $15(4.1)$ & $10(13.5)$ & $5(1.7)$ & $<.001^{\mathrm{d}}$ \\
\hline Anxiolytics & $31(8.4)$ & $12(16.2)$ & $19(6.5)$ & $.007^{\mathrm{d}}$ \\
\hline \multicolumn{5}{|l|}{ Cardiac rehabilitation components ${ }^{\mathrm{e}}$} \\
\hline Introduction & $144(44.4)$ & $29(45.3)$ & $115(44.2)$ & .88 \\
\hline Risk factors & $251(77.0)$ & $46(70.8)$ & $205(78.5)$ & .18 \\
\hline Nutrition/Dietary advice & $238(73.0)$ & $43(66.2)$ & $195(74.7)$ & .16 \\
\hline Medication & $196(59.0)$ & $38(57.6)$ & $158(59.4)$ & .79 \\
\hline Physical exercise & $327(98.5)$ & $64(97.0)$ & $263(98.9)$ & .26 \\
\hline Stress management & $61(18.4)$ & 8 (1214) & $53(20.0)$ & .14 \\
\hline Smoking cessation & $26(7.8)$ & $4(6.1)$ & $22(8.3)$ & .55 \\
\hline Weight loss & $53(16.0)$ & $11(16.7)$ & $42(15.8)$ & .86 \\
\hline Individual counseling & $48(14.5)$ & $14(21.2)$ & $34(12.8)$ & .08 \\
\hline
\end{tabular}

${ }^{\text {a }}$ Results are presented as $n(\%)$ unless otherwise stated

b CABG, MI, or PCI

${ }^{c} \mathrm{COPD}=$ Chronic obstructive pulmonary disease

d $P<.05$

e Due to missing data for 36-44 patients, analyses were conducted on available data

covariates, somatic and cognitive symptoms decreased during the 3-month follow-up period $(F(1,357)=3.75$, $P=.05)$. The interaction effect for time by anhedonia remained significant after controlling for covariates $(F(1,357)=3.86, P=.05)$. Likewise, the main betweensubjects effect for anhedonia remained significant $(F(1,357)=13.00, P<.001)$. The main between-subjects effects for the HADS Negative Affect scale $(F(1,357)=$ 123.46, $P<.001$ ) were significant.

Post hoc analyses showed that anhedonic patients reported a decrease in somatic as well as cognitive symptoms following $\mathrm{CR}(t(73)=5.48$ and $t(293)=5.22$, both
$P$ s $<.001)$. Likewise, non-anhedonic patients also reported a decrease in somatic and cognitive symptoms after attending the CR program $(t(293)=7.07$ and $t(293)=$ 6.54), both $P$ s $<.001)$.

\section{Discussion}

Studies on the role of psychological factors in CR programs have merely focused on the role of negative affect. In contrast, little is known about the effects of positive affect in CAD. To the best of our knowledge, this is the 
Table 2 Factor structure of the HADS ${ }^{\mathrm{a}}$

\begin{tabular}{|c|c|c|c|c|}
\hline & HADS Items & $\begin{array}{l}\text { Factor } \mathrm{I}^{\mathrm{b}} \\
\text { Positive affect }\end{array}$ & $\begin{array}{l}\text { Factor } \text { II }^{\mathrm{b}} \\
\text { Negative affect }\end{array}$ & $\begin{array}{l}\text { Factor } \text { III }^{\mathrm{b}} \\
\text { Relaxed affect }\end{array}$ \\
\hline 6 & I feel cheerful & .73 & -.20 & .27 \\
\hline 2 & I still enjoy the things I used to enjoy & .69 & & .24 \\
\hline 4 & I can laugh and see the funny side of things & .72 & -.24 & .30 \\
\hline 12 & I look forward with enjoyment to things & .73 & & \\
\hline 3 & I get a sort of frightened feeling as if something awful is about to happen & & .81 & \\
\hline 13 & I get sudden feelings of panic & & .75 & \\
\hline 5 & Worrying thoughts go through my mind & -.37 & .69 & -.25 \\
\hline 1 & I feel tense or wound up & -.42 & .54 & -.30 \\
\hline 7 & I can sit at ease and feel relaxed & -.33 & -.38 & .64 \\
\hline 14 & I can enjoy a good book or TV program & .31 & & .66 \\
\hline \multirow[t]{2}{*}{11} & I feel restless as if I have to be on the move & & & .81 \\
\hline & Internal consistency (Cronbach's $\alpha)$ & .83 & .81 & 67 \\
\hline
\end{tabular}

HADS $=$ Hospital Anxiety Depression Scale

${ }^{\text {a }}$ Loadings $<.20$ are not displayed

b Items assigned to a factor are presented in bold-face

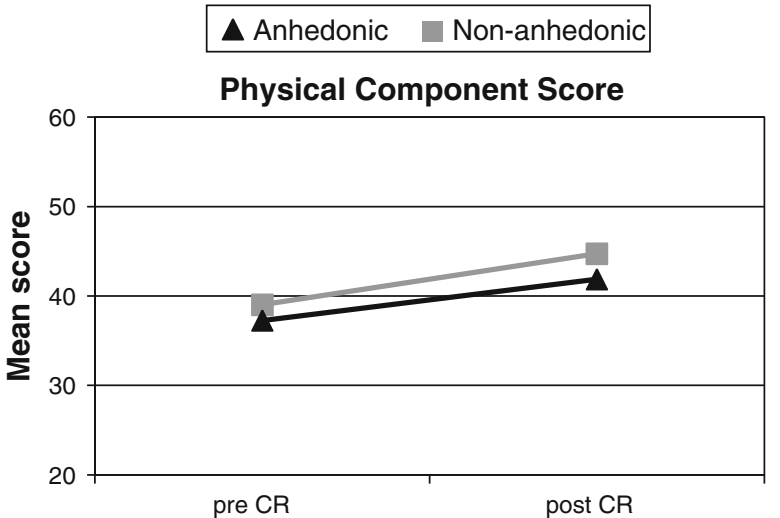

Mental Component Score

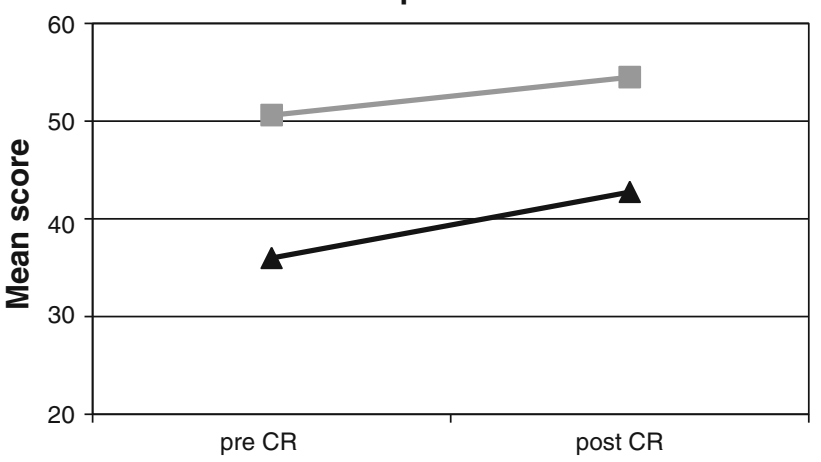

Fig. 2 Mean health status scores pre- and post-CR stratified by anhedonia. MANOVA with repeated measures. A high score represents better health status. Note: SDs range from 6.94 to 11.24. Main effect for Time $F(1,357)=10.84, P=.001$; Main effect for Anhedonia $F(1,357)=34.80, P<.001$; Interaction for Time*Anhedonia $F(1,357)=.23, P=.63$. $C R$ Cardiac rehabilitation
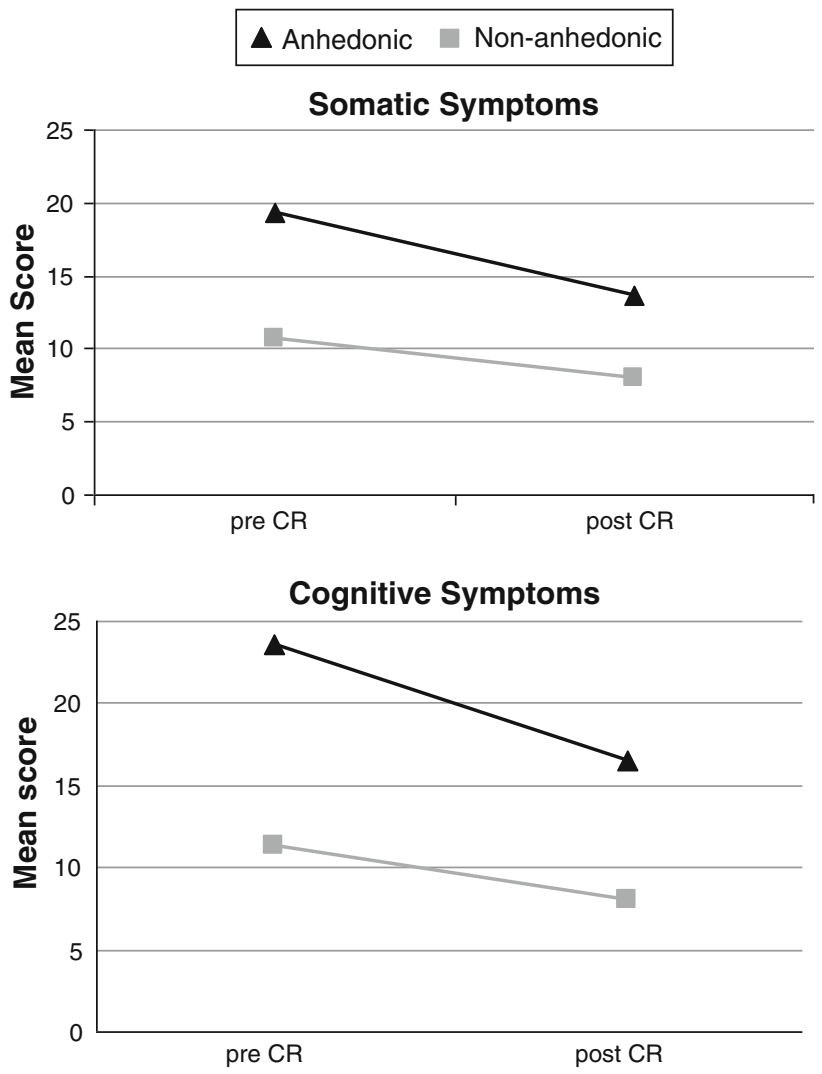

Fig. 3 Mean somatic and cognitive symptoms pre- and post-CR stratified by anhedonia. MANOVA with repeated measures. Note: SDs range from 7.40 to 12.73 . Main effect for Time $F(1,357)=3.75$, $P=.05$; Main effect for Anhedonia $F(1,357)=13.00, P<.001$; Interaction for Time*Anhedonia $F(1,357)=3.86, P=.05 . C R$ Cardiac rehabilitation 
first study to demonstrate that anhedonic patients, i.e. the lack of positive affect, reported more impaired health status and higher levels of health complaints prior to and after CR attendance compared with non-anhedonic patients. Furthermore, the current study pointed out that patients' health status improved and somatic and cognitive symptoms decreased in both anhedonic as well as non-anhedonic patients over time. In addition, we found an interaction effect for anhedonia by time for somatic and cognitive symptoms, indicating that anhedonic patients reported more benefit from $\mathrm{CR}$ in terms of reduction in somatic and cognitive symptoms. The interaction effect for time by health status was not significant.

Our study was in line with previous findings, showing that CR may improve health status [2] and diminish somatic and cognitive symptoms [28]. However, in the present study, we were also able to identify a specific subgroup of patients - namely anhedonic patients-who consistently reported impaired health status and higher levels of somatic and cognitive symptoms despite CR attendance. The importance of anhedonia has been demonstrated previously in CAD, with anhedonia being a risk factor for major clinical adverse events following implantation of coronary-artery stents [18], and the combined endpoint of adverse clinical events and all-cause mortality [17]. The present study elaborates on these findings by showing that in a large sample of CR patients, patientcentered outcomes vary as a function of the level of anhedonia as well.

In addition, in this study, we replicated findings on the underlying factorial structure of the HADS. Originally, this instrument was developed to assess depressive and anxious symptomatology in hospitalized patients [20, 21]. However, two recent studies suggest that it is also possible to derive a measure of anhedonia from the $\operatorname{HADS}[18,34]$. Hence, with the HADS, it is possible to tap into several psychological constructs that have been shown to impact on patient well-being and prognosis in CAD without increasing patient burden, making it an opportune instrument to use in clinical practice. In line with these two other studies, we found that HADS assesses Negative Affect, Relaxed Affect, and Positive Affect. The construct validity of these subscales was confirmed by the significant medium to large correlations with the GMS, an instrument that previously has been shown to valid and reliably assess positive and negative affect [22-24]. Furthermore, the three derived HADS scales were shown to be internally consistent $(.83<\alpha<.67)$.

The notion of positive and negative affect not just merely being the opposite two ends of a continuum [10], and the possibility that both types of affect can be present simultaneously, broadens the scope. The present study supports this notion, as the effects of anhedonia remained significant after controlling for the confounding effects of negative affect. The combined effects of negative and positive affect, i.e. the interaction between those two types of affect, might refine findings and contribute to a fuller understanding of the role of affect in the context of CAD.

Limitations of the current study must be acknowledged. First, we were not able to control for markers of disease severity, (e.g. left ventricular ejection fraction) as these were not consistently collected in the current study. Moreover, in the present study, we only evaluated the effect of anhedonia on short-term patient-centered outcomes. Whether these improvements remain over time is unknown, but it has been shown that the effects of CR on health status remain over time [35, 36]. Further, patients diagnosed with chronic heart failure were excluded in the current study, due to participation in another study. Results from the current study can therefore not be generalized to this specific patient group. Fourth, information on psychiatric diagnoses and objective outcomes, such as exercise capacity, are lacking. Finally, the present study was based on a between-subjects design and improvements in health outcomes cannot be attributed to $\mathrm{CR}$, due to the lack of a control-group. Strengths of the study comprise the large sample size and the use of valid and reliable instruments to assess patient-centered outcomes. In addition, both generic as well as disease-specific questionnaires were administered to evaluate $\mathrm{CR}$ in CAD patients.

From a clinical point of view, studies on anhedonia pave the way for the development of new interventions for secondary prevention. Positive affect has been shown to be associated with biological indices of cardiac disease in healthy individuals, like salivary cortisol, systolic blood pressure, and inflammatory markers [37-39]. However, up to now, most studies have focused on the detrimental effects of negative affective states, like distress [8] and depressive symptoms [6] on CR outcome. Our results indicate that anhedonia is also of importance in the context of CR. Consequently, incorporating and encouraging the development of skills to experience more positive affect might contribute to increased benefits from CR programs. Cognitive-behavioral therapy and mindfulness-based stress reduction have been shown to improve positive affect in medically ill patients [40, 41], and in older depressed patients at increased cardiovascular risk [42], and might be effective in enhancing positive affect in patients attending CR.

Future studies are warranted to further determine the role of anhedonia on hard outcomes like (re-)hospitalization, major clinical adverse events (MACE), and survival. Anhedonia, or the lack of positive affect, independently predicted major clinical adverse events following implantation of coronary-artery stents [18], and the combined endpoint of MACE and all-cause mortality in post-MI 
patients [17] in previous studies. However, the current study is of importance as health status has been shown to predict mortality in CAD patients [43], and patient-centered outcomes and the identification of its determinants have been advocated to bridge the gap between research and clinical practice [19].

In conclusion, the present study showed that anhedonic CAD patients reported poorer health status and higher levels of somatic and cognitive symptoms prior to and after $\mathrm{CR}$ in comparison with non-anhedonic patients. Furthermore, health status improved and somatic and cognitive symptoms decreased in both anhedonic and non-anhedonic patients. Somatic and cognitive symptoms pre- and postCR changed differentially for anhedonic and non-anhedonic patients, with anhedonic patients reporting more changes in somatic and cognitive symptoms. These findings underscore the importance of studying positive affect within the context of CR.

Acknowledgments The authors would like to thank Eva Pruyn, Marise C. Van Linden, Anne van Schijndel, and Michiel Vollenbronck for assistance with data collection. This research was in part supported by the Netherlands Organization for Scientific Research (NWO) with a VENI grant (451-05-001) to S.S. Pedersen and a VICI grant (453-04-004) to J. Denollet.

\section{Conflicts of interest None.}

Open Access This article is distributed under the terms of the Creative Commons Attribution Noncommercial License which permits any noncommercial use, distribution, and reproduction in any medium, provided the original author(s) and source are credited.

\section{References}

1. Williams, M. A., Ades, P. A., Hamm, L. F., Keteyian, S. J., LaFontaine, T. P., Roitman, J. L., et al. (2006). Clinical evidence for a health benefit from cardiac rehabilitation: An update. American Heart Journal, 152, 835-841.

2. Cohen, R. A., Moser, D. J., Clark, M. M., Aloia, M. S., Cargill, B. R., Stefanik, S., et al. (1999). Neurocognitive functioning and improvement in quality of life following participation in cardiac rehabilitation. American Journal of Cardiology, 83, 1374-1378.

3. Taylor, R. S., Unal, B., Critchley, J. A., \& Capewell, S. (2006). Mortality reductions in patients receiving exercise-based cardiac rehabilitation: How much can be attributed to cardiovascular risk factor improvements? European Journal of Cardiovascular Prevention and Rehabilitation, 13, 369-374.

4. Verges, B., Patois-Verges, B., Cohen, M., Lucas, B., Galland-Jos, C., \& Casillas, J. M. (2004). Effects of cardiac rehabilitation on exercise capacity in Type 2 diabetic patients with coronary artery disease. Diabetic Medicine, 21, 889-895.

5. Lavie, C. J., \& Milani, R. V. (1999). Effects of cardiac rehabilitation and exercise training programs on coronary patients with high levels of hostility. Mayo Clinic Proceedings, 74, 959-966.

6. Milani, R. V., \& Lavie, C. J. (2007). Impact of cardiac rehabilitation on depression and its associated mortality. American Journal of Medicine, 120, 799-806.
7. Lavie, C. J., \& Milani, R. V. (2006). Impact of cardiac rehabilitation on depression and its associated mortality. American Journal of Medicine, 120, 799-806.

8. Hevey, D., McGee, H. M., \& Horgan, J. (2007). Relationship of initial level of distress to changes in health-related quality of life during cardiac rehabilitation or usual care. Psychosomatic Medicine, 69, 793-797.

9. Pressman, S. D., \& Cohen, S. (2005). Does positive affect influence health? Psychological Bulletin, 131, 925-971.

10. Larsen, J. T., McGraw, A. P., \& Cacioppo, J. T. (2001). Can people feel happy and sad at the same time? Journal of Personality and Social Psychology, 81, 684-696.

11. Kubzansky, L. D., \& Thurston, R. C. (2007). Emotional vitality and incident coronary heart disease: Benefits of healthy psychological functioning. Archives of General Psychiatry, 64, 1393-1401.

12. Middleton, R., \& Byrd, K. (1996). Psychosocial factors and hospital readmission status of older persons with cardiovascular disease. Journal of Applied Rehabilitation Counseling, 27, 3-10.

13. Konstam, V., Salem, D., Pouleur, H., Kostis, J., Gorkin, L., Shumaker, S., et al. (1996). Baseline quality of life as a predictor of mortality and hospitalization in 5, 025 patients with congestive heart failure. SOLVD investigations. Studies of left ventricular dysfunction investigators. American Journal of Cardiology, 78, 890-895.

14. Rumsfeld, J. S., MaWhinney, S., McCarthy, M., Shroyer, A. L., VillaNueva, C. B., O’Brien, M., et al. (1999). Health-related quality of life as a predictor of mortality following coronary artery bypass graft surgery. Participants of the Department of Veterans Affairs Cooperative Study Group on Processes, Structures, and Outcomes of Care in Cardiac Surgery. Journal of the American Medical Association, 281, 1298-1303.

15. Van Domburg, R. T., Pedersen, S. S., van den Brand, M. J., \& Erdman, R. A. (2001). Feelings of being disabled as a predictor of mortality in men 10 years after percutaneous coronary transluminal angioplasty. Journal of Psychosomatic Research, 51, 469-477.

16. Kubzansky, L. D., Sparrow, D., Vokonas, P., \& Kawachi, I. (2001). Is the glass half empty or half full? A prospective study of optimism and coronary heart disease in the Normative Aging Study. Psychosomatic Medicine, 63, 910-916.

17. Davidson, K. W., Burg, M. M., Kronish, I. M., Shimbo, D., Dettenborn, L., Mehran, R., et al. (2010). Association of anhedonia with recurrent major adverse cardiac events and mortality 1 year after acute coronary syndrome. Archives General Psychiatry, 67, 480-488.

18. Denollet, J., Pedersen, S. S., Daemen, J., de Jaegere, P., Serruys, P. W., \& van Domburg, R. T. (2008). Reduced positive affect (anhedonia) predicts major clinical events following implantation of coronary-artery stents. Journal of Internal Medicine, 263, 203-211.

19. Krumholz, H. M., Peterson, E. D., Ayanian, J. Z., Chin, M. H., DeBusk, R. F., Goldman, L., et al. (2005). Report of the National Heart, Lung, and Blood Institute working group on outcomes research in cardiovascular disease. Circulation, 111, 3158-3166.

20. Zigmond, A. S., \& Snaith, R. P. (1983). The hospital anxiety and depression scale. Acta Psychiatrica Scandinavica, 67, 361-370.

21. Spinhoven, P., Ormel, J., Sloekers, P. P., Kempen, G. I., Speckens, A. E., \& van Hemert, A. M. (1997). A validation study of the Hospital Anxiety and Depression Scale (HADS) in different groups of Dutch subjects. Psychological Medicine, 27, 363-370.

22. Denollet, J. (1993). Emotional distress and fatigue in coronary heart disease: The Global Mood Scale (GMS). Psychological Medicine, 23, 111-121.

23. Denollet, J. (1993). Sensitivity of outcome assessment in cardiac rehabilitation. Journal of Consulting and Clinical Psychology, 61, 686-695. 
24. Denollet, J., \& De Vries, J. (2006). Positive and negative affect within the realm of depression, stress and fatigue: The two-factor distress model of the Global Mood Scale (GMS). Journal of Affective Disorders, 91, 171-180.

25. Ware, J. E., Jr. (1993). SF-36 health survey: Manual and interpretation guide. Boston: The Health Institute, New England Medical Centre.

26. Aaronson, N. K., Muller, M., Cohen, P. D., Essink-Bot, M. L., Fekkes, M., Sanderman, R., et al. (1998). Translation, validation, and norming of the Dutch language version of the SF-36 Health Survey in community and chronic disease populations. Journal of Clinical Epidemiology, 51, 1055-1068.

27. Ware, J. E., \& Kosinski, M. (2001). Interpreting SF-36 summary health measures: A response. Quality of Life Research, 10, 405-413.

28. Denollet, J. (1994). Health complaints and outcome assessment in coronary heart disease. Psychosomatic Medicine, 56, 463-474.

29. Pedersen, S. S., \& Denollet, J. (2002). Perceived health following myocardial infarction: Cross-validation of the Health Complaints Scale in Danish patients. Behaviour Research and Therapy, 40, 1221-1230.

30. McGee, H. M., Hevey, D., \& Horgan, J. H. (1999). Psychosocial outcome assessments for use in cardiac rehabilitation service evaluation: A 10-year systematic review. Social Science and Medicine, 48, 1373-1393.

31. Freedland, K. E., Babyak, M. A., McMahon, R. J., Jennings, J. R., Golden, R. N., \& Sheps, D. S. (2005). Statistical guidelines for psychosomatic medicine. Psychosomatic Medicine, 67, 167.

32. Aalto, A. M., Aro, A. R., Weinman, J., Heijmans, M., Manderbacka, K., \& Elovainio, M. (2006). Sociodemographic, disease status, and illness perceptions predictors of global self-ratings of health and quality of life among those with coronary heart disease-one year follow-up study. Quality of Life Research, 15, $1307-1322$.

33. Pelle, A. J., Erdman, R. A., van Domburg, R. T., Spiering, M., Kazemier, M., \& Pedersen, S. S. (2008). Type D patients report poorer health status prior to and after cardiac rehabilitation compared to non-Type D patients. Annals of Behavioral Medicine, 36, 167-175.

34. Martin, C. R., Lewin, R. J., \& Thompson, D. R. (2003). A confirmatory factor analysis of the Hospital Anxiety and Depression
Scale in coronary care patients following acute myocardial infarction. Psychiatry Research, 120, 85-94.

35. Gupta, R., Sanderson, B. K., \& Bittner, V. (2007). Outcomes at one-year follow-up of women and men with coronary artery disease discharged from cardiac rehabilitation: What benefits are maintained? Journal of Cardiopulmonary Rehabilitation and Prevention, 27, 11-18.

36. Yu, C. M., Li, L. S., Ho, H. H., \& Lau, C. P. (2003). Long-term changes in exercise capacity, quality of life, body anthropometry, and lipid profiles after a cardiac rehabilitation program in obese patients with coronary heart disease. American Journal of Cardiology, 91, 321-325.

37. Polk, D. E., Cohen, S., Doyle, W. J., Skoner, D. P., \& Kirschbaum, C. (2005). State and trait affect as predictors of salivary cortisol in healthy adults. Psychoneuroendocrinology, 30, 261-272.

38. Steptoe, A., Wardle, J., \& Marmot, M. (2005). Positive affect and health-related neuroendocrine, cardiovascular, and inflammatory processes. Proceedings of the National Academy of Science, 102, 6508-6512.

39. Steptoe, A., O’Donnell, K., Badrick, E., Kumari, M., \& Marmot, M. (2008). Neuroendocrine and inflammatory factors associated with positive affect in healthy men and women: The Whitehall II study. American Journal of Epidemiology, 167, 96-102.

40. Grossman, P., Tiefenthaler-Gilmer, U., Raysz, A., \& Kesper, U. (2007). Mindfulness training as an intervention for fibromyalgia: Evidence of postintervention and 3-year follow-up benefits in well-being. Psychotherapy and Psychosomatics, 76, 226-233.

41. Mohr, D. C., Hart, S. L., Julian, L., Catledge, C., Honos-Webb, L., Vella, L., et al. (2005). Telephone-administered psychotherapy for depression. Archives of General Psychiatry, 62, 1007-1014.

42. Strachowski, D., Khaylis, A., Conrad, A., Neri, E., Spiegel, D., \& Taylor, C. B. (2008). The effects of cognitive behavior therapy on depression in older patients with cardiovascular risk. Depression and Anxiety, 25, e1-e10.

43. Lenzen, M. J., Scholte op Reimer, W. J., Pedersen, S. S., Boersma, E., Maier, W., Widimsky, P., et al. (2007). The additional value of patient-reported health status in predicting 1-year mortality after invasive coronary procedures: A report from the Euro Heart Survey on Coronary Revascularisation. Heart, 93, $339-344$ 